\title{
Gamificação em Ambientes Educacionais Ubíquos
}

\author{
Hiran Nonato M. Ferreira ${ }^{1,2}$, Rafael D. Araújo ${ }^{1}$, Paula C. Souza ${ }^{1}$, \\ Samuel Chagas da S. Júnior ${ }^{1}$, Fabiano A. Dorça ${ }^{1}$, Renan G. Cattelan ${ }^{1}$ \\ ${ }^{1}$ Faculdade de Computação - Universidade Federal de Uberlândia \\ Caixa Postal 593 - CEP 38408-100 - Uberlândia - MG - Brasil \\ ${ }^{2}$ Instituto Federal do Sul de Minas Gerais - IFSULDEMINAS \\ Rua Mário Ribola, 409 - CEP 37903-358 - Passos - MG - Brasil \\ ubimedia@facom.ufu.br
}

\begin{abstract}
One challenge in educational computing environments is to ensure the student engagement and motivation in using their resources. In this sense, gamification concepts have been employed to adapt game elements to learningteaching activities in order to make them more engaging and attractive. This paper presents the process of design and development of a software module for gamification in Ubiquitous Learning Environments (ULEs). Besides presenting the proposed module's architecture and its implementation details, we describe its integration to a real ULE application and its use together with collaborative mechanisms for the extension and enrichment of educational content.
\end{abstract}

Resumo. Um dos desafios dos ambientes computacionais voltados à educação é garantir o engajamento e manter a motivação dos estudantes em sua utilização. Nesse sentido, conceitos de gamificação têm sido explorados para adaptar elementos de jogos a atividades de ensino e aprendizagem no intuito de torná-las mais envolventes e atrativas. Este artigo apresenta o processo de concepção e desenvolvimento de um módulo de software para gamificação de Ambientes Educacionais Ubíquos (AEUs). Além da arquitetura do módulo de gamificação proposto e dos detalhes técnicos de sua implementação, é descrita sua integração a um AEU real e sua utilização conjunta com mecanismos colaborativos de extensão e enriquecimento de conteúdo educacional.

\section{Introdução}

Cada vez mais, recursos computacionais têm sido integrados a ambientes educacionais com o intuito de melhorar a dinâmica de ensino-aprendizagem. Uma das propostas para apoiar atividades em sala de aula consiste no uso de Ambientes Educacionais Ubíquos (AEUs) [Settle et al. 2011] - espaços físicos equipados com recursos computacionais (sensores, lousas eletrônicas, câmeras, microfones, entre outros) com o potencial de incorporar a visão de computação ubíqua [Weiser 1991] à área de educação.

Quando associados a sistemas automatizados de captura multimídia, os AEUs permitem o registro e o armazenamento de artefatos de mídia que, uma vez combinados e sincronizados, são capazes de reproduzir posteriormente as experiências vivenciadas em sala de aula. Nesses ambientes, os estudantes podem centrar a atenção na experiência da aula, com a confiança de que os detalhes estão sendo registrados e estarão disponíveis para acesso futuro. 
Diversas pesquisas com AEUs estão voltadas para a fase de acesso, momento em que as informações capturadas são recuperadas e apresentadas aos usuários [Abowd e Mynatt 2000, Kientz 2012, Araujo et al. 2013, Mendonca et al. 2014]. Nessa fase, os formatos e as interfaces de apresentação devem ser intuitivos e permitir mais que o simples acesso às informações, provendo conteúdo de modo dinâmico e tornando possível a interação com outros usuários (instrutores e estudantes) a fim de compartilhar e enriquecer o conteúdo originalmente capturado. Para tanto, é essencial manter os usuários engajados e motivados no uso dessas plataformas de ensino.

De acordo com Kiili [Kiili et al. 2012] uma das limitações intrínsecas aos processos de aprendizagem em ambientes computacionais voltados à educação está relacionada justamente ao engajamento dos estudantes, que muitas vezes não se sentem estimulados a utilizarem esses ambientes. Uma proposta que busca contornar esse comportamento é a incorporação de elementos e mecanismos de jogos incorporados ao ambiente computacional, visando torná-lo mais atrativo aos estudantes e alavancar sua utilização. Essa abordagem é conhecida como gamificação (do Inglês, gamification) [Seaborn e Fels. 2014]. A inserção de elementos de jogos a contextos não lúdicos tem o potencial de gerar, entre os participantes, um nível de envolvimento semelhante ao que é conseguido com os jogos [Deterding et al. 2011]. Segundo Lee e Hammer [Lee e Hammer 2011], o objetivo dessa abordagem não é ensinar com os jogos, mas sim usar elementos de jogos como forma de promover a motivação e o envolvimento dos estudantes.

Este artigo apresenta o processo de concepção e desenvolvimento de um módulo de software para gamificação de AEUs. Além da arquitetura do módulo e dos detalhes técnicos de sua implementação, é descrita sua integração a um AEU real e sua utilização com mecanismos colaborativos de extensão e enriquecimento de conteúdo educacional. A arquitetura proposta utiliza diversos mecanismos de competição (pontuação, rankings, conquista de medalhas, entre outros) para melhorar a dinâmica de utilização do ambiente.

O restante do artigo está estruturado da seguinte maneira: na Seção 2, é apresentado o Classroom eXperience, o AEU que foi utilizado como estudo de caso para integração do módulo de gamificação proposto; na Seção 3, são detalhados os componentes e os elementos que compõem a arquitetura do modelo de gamificação; na Seção 4, é apresentado o protótipo desenvolvido, bem como a sua integração ao AEU; na Seção 5, são discutidos trabalhos relacionados; e, por fim, na Seção 6, são apresentadas as considerações finais e potenciais trabalhos futuros.

\section{Classroom eXperience}

O Classroom eXperience (CX) [Ferreira et al. 2012, Araujo et al. 2013] é uma plataforma multimídia para captura de aulas em uma sala instrumentada com dispositivos computacionais ubíquos, tais como lousa eletrônica, microfones, câmeras de vídeo e projetores. Desenvolvido para registrar, armazenar, sincronizar e disponibilizar as diferentes mídias capturadas, o CX gera documentos hipermídia em diferentes formatos de apresentação e que podem ser utilizados para reconstituir a experiência vivida em sala de aula.

Por se tratar de uma plataforma baseada conceitos de AEUs, o CX realiza a captura da aula de maneira automática, transparente e não intrusiva, empregando recursos de aplicações de Captura e Acesso (C\&A) para auxiliar tanto instrutores, durante o processo de captura, quanto estudantes, no acesso ao conteúdo capturado. O sistema conta com um 
arcabouço de hardware e software especializado para realizar a gravação das atividades educacionais, sincronizar os fluxos de mídia e disponibilizar o conteúdo por meio de uma interface de acesso amigável e intuitiva. A Figura 1 apresenta (a) uma sala de aula instrumentada utilizada pelo CX e (b) um exemplo de documento de uma aula capturada.

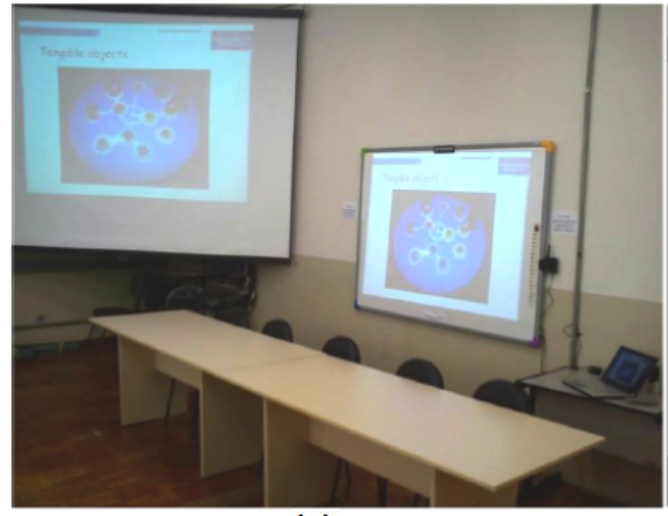

(a)

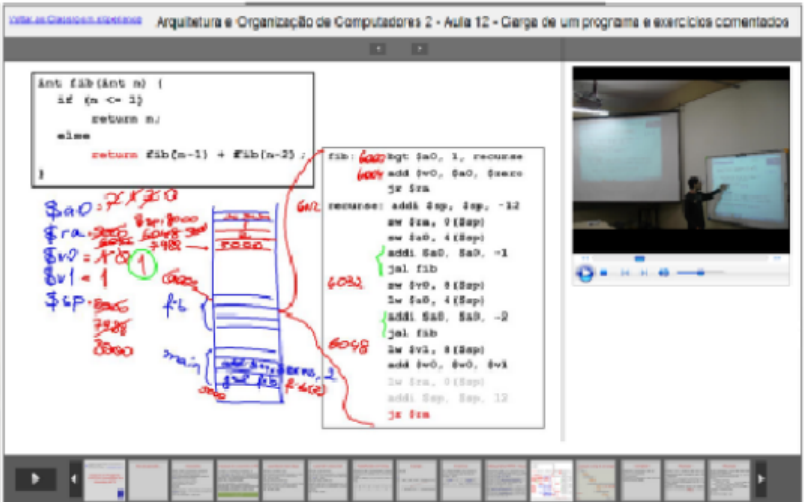

(b)

Figura 1. Classroom eXperience: (a) sala instrumentada utilizada para captura de aulas; (b) aula capturada com anotações e vídeo, disponibilizado para visualização no formato HTML [Mendonca et al. 2014].

Concebida como uma aplicação de C\&A, o CX implementa o modelo proposto por [Truong e Hayes 2009], composto de quatro fases distintas: (1) pré-produção, que consiste na preparação do conteúdo para captura; (2) gravação "ao vivo", em que múltiplos fluxos de informação são capturados de dispositivos espalhados pelo ambiente; (3) pós-produção, em que os fluxos capturados na fase anterior são sincronizados e integrados; e (4) acesso, responsável por disponibilizar aos usuários o conteúdo capturado.

Além de permitir acesso às informações capturadas de forma simplificada, o CX possui a capacidade de personalizar o conteúdo a ser apresentado com base nas preferências e no contexto de acesso de cada usuário. Para isso, o CX utiliza módulos de personalização de conteúdo baseados em preferências, contexto e restrições de apresentação [Araujo et al. 2013]. Sua arquitetura permite ainda a extensão e o enriquecimento de conteúdo por meio de mecanismos de anotação e classificação (ranqueamento) disponibilizados aos estudantes [Mendonca et al. 2014]. A Figura 2 apresenta uma das interfaces de acesso para visualização do conteúdo capturado, permitindo a criação de anotações e o ranqueamento da informação apresentada.

\section{Módulo de Gamificação}

A metodologia de pesquisa na área de computação ubíqua é baseada na experimentação com protótipos e na validação de abstrações e modelos [Weiser 1991]. Durante esta pesquisa foram desenvolvidos protótipos funcionais em quantidade suficiente para depurar a viabilidade da proposta. Assim, este artigo teve como foco o desenvolvimento de um protótipo para experimentação de funcionalidades de gamificação em AEUs. O principal objetivo da gamificação no contexto educacional é a utilização de mecânicas de jogos que tornam o processo educativo mais interessante e atrativo [Domínguez et al. 2013]. A abordagem proposta não busca a criação de um jogo específico, e sim a utilização da gamificação para tornar AEUs mais atraentes e divertidos, aumentando seu uso. 


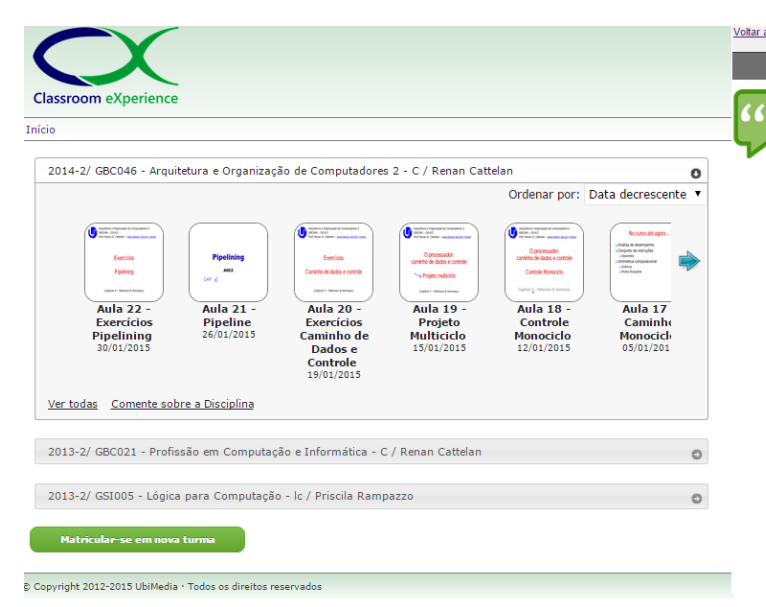

(a)

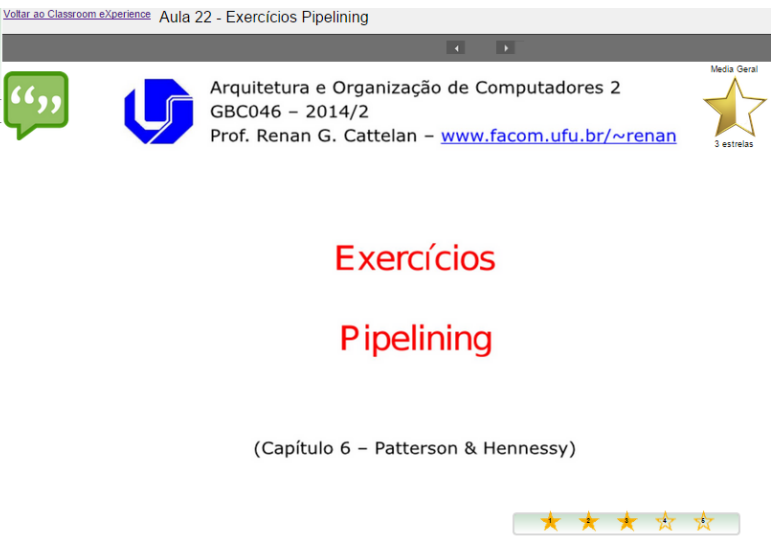

(b)

Figura 2. Classroom eXperience: (a) Interface para acesso às aulas capturadas; (b) Interface para visualização, sendo possível realizar anotações e ranqueamento.

O módulo de gamificação desenvolvido para o CX dispõe de diversos mecanismos de jogos (pontuação, rankings, conquista de medalhas, assiduidade de uso do sistema, entre outros) com o objetivo de proporcionar um maior engajamento dos estudantes na utilização da plataforma. Esses mecanismos concentram-se em torno da pontuação do usuário, que é conquistada através de suas interações com o sistema. Cada interação possui um peso específico, conforme sua relevância durante a utilização do sistema.

Os usuários se empenham no desenvolvimento de alguma atividade quando percebem que são recompensados por isso. Um mecanismo de pontuação é uma boa forma de motivar e proporcionar um maior engajamento dos usuários a uma determinada atividade [Lee e Hammer 2011]. Dessa forma, o módulo de gamificação proposto atribui pontos às ações dos usuários com o intuito de classificá-los no universo de estudantes. Todas as atividades de extensão e enriquecimento de conteúdo são pontuadas. Existem quatro formas de se conquistar pontos:

1. Cada vez que o estudante realiza login no sistema - 0,5 ponto;

2. Sempre que o estudante classifica uma aula ou um slide como importante (utilizando mecanismos de ranqueamento por estrelas) - 1,0 ponto;

3. Sempre que o estudante comenta uma aula ou um slide - 2,0 pontos;

4. Sempre que o estudante responde corretamente a uma pergunta disponibilizada pelo instrutor (quiz) $-4,0$ pontos.

Todas essas formas de pontuação são utilizadas para motivar o estudante a utilizar as diferentes funcionalidades disponibilizadas pelo sistema. Sempre que o usuário realiza login no sistema é verificado o horário do último acesso, caso seja inferior a um tempo pré-determinado, o usuário não pontuará. A Figura 3 ilustra partes da interface com as quais o usuário pode interagir para acumular pontos.

O módulo de gamificação classifica os estudantes de acordo com o seu desempenho. Conforme a dinâmica de competição, podem ser utilizados critérios de competição externa (quando o estudante compete com outros usuários) quanto critérios de autocompetição (quando o estudante compete consigo mesmo) para formar três ranks: 


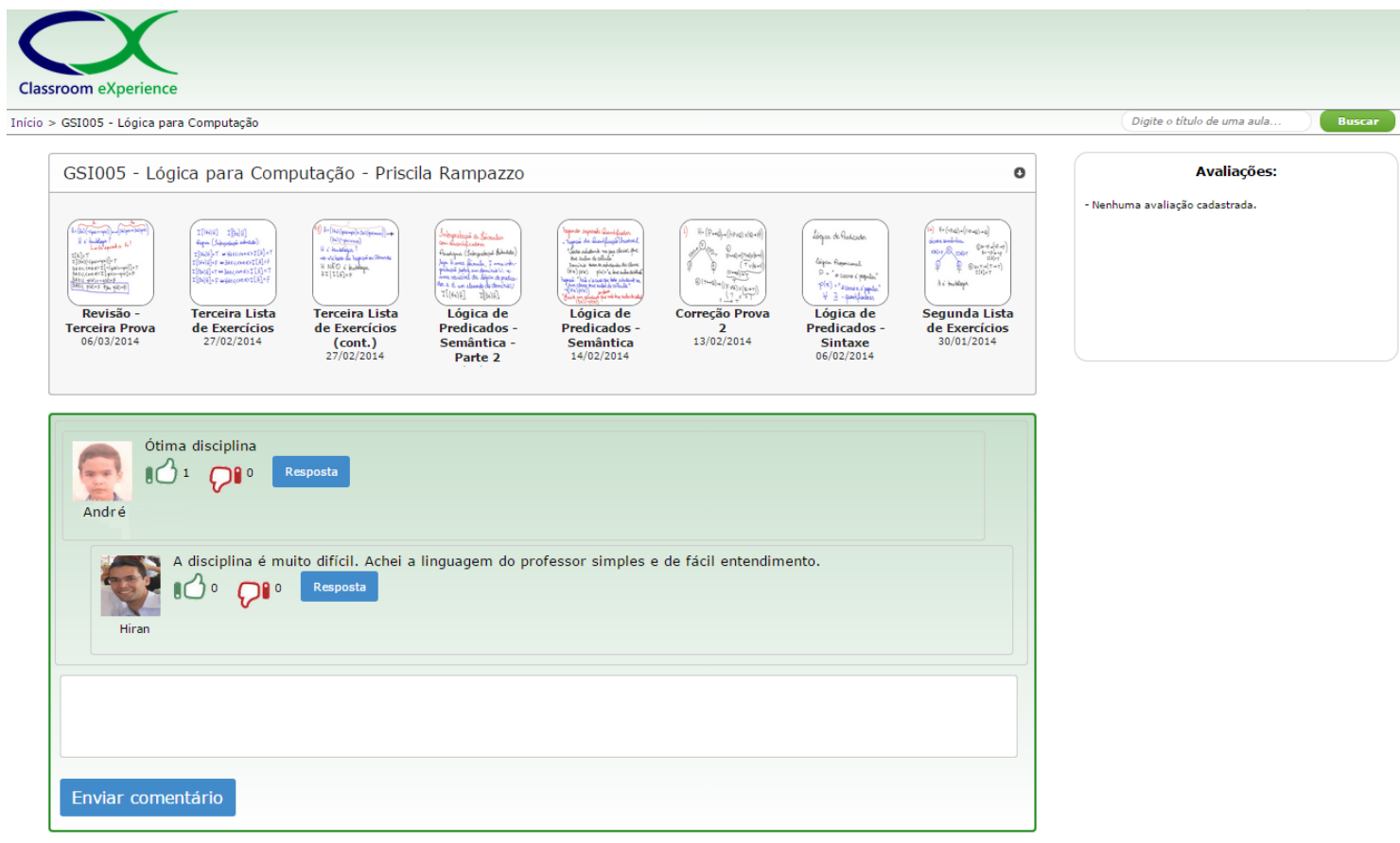

Figura 3. Interface do CX com suporte a gamificação: comentário sobre a disciplina permite acúmulo de pontos.

- Ranking geral - é utilizado um quadro de classificação geral mostrando os dez melhores estudantes de todo o sistema. Esse quadro é apresentado na tela inicial, à qual todos os estudantes têm acesso. O ranking geral é independente da disciplina e do nível do curso do estudante, sendo que todos competem contra todos.

- Ranking por disciplina - é utilizado um quadro que exibe os melhores estudantes dentro de uma disciplina. Este quadro é apresentado na página de cada disciplina. No ranking por disciplina, os estudantes competem com os colegas inscritos naquela disciplina, sem considerar pontos adquiridos em outras.

- Conquista de medalhas - é um mecanismo de autocompetição, onde os estudantes buscam alcançar uma quantidade de pontos para conseguir símbolos de recompensa. Nesse mecanismo, o estudante ganha um determinado distintivo, dependendo da pontuação que atinge (300 pontos: distintivo de nível iniciante; 500 pontos: distintivo de nível intermediário; 1000 pontos: distintivo de nível avançado).

A Figura 4 apresenta a interface principal de acesso de uma disciplina. Na parte direita, é possível notar a presença do ranking por disciplina e as medalhas conquistadas.

Além das interfaces de acesso para os estudantes, o módulo de gamificação também permite acompanhamento da pontuação pelo instrutor da disciplina. O instrutor tem acesso ao desempenho dos seus alunos a partir das suas respectivas pontuações e conquistas. Com isso, é possível acompanhar quem realmente está interagindo de forma mais efetiva no ambiente de aprendizagem.

\section{Arquitetura e Implementação}

O protótipo responsável pelo gerenciamento e controle dos componentes de gamificação foi integrado ao CX através de uma infraestrutura fracamente acoplada, buscando a 


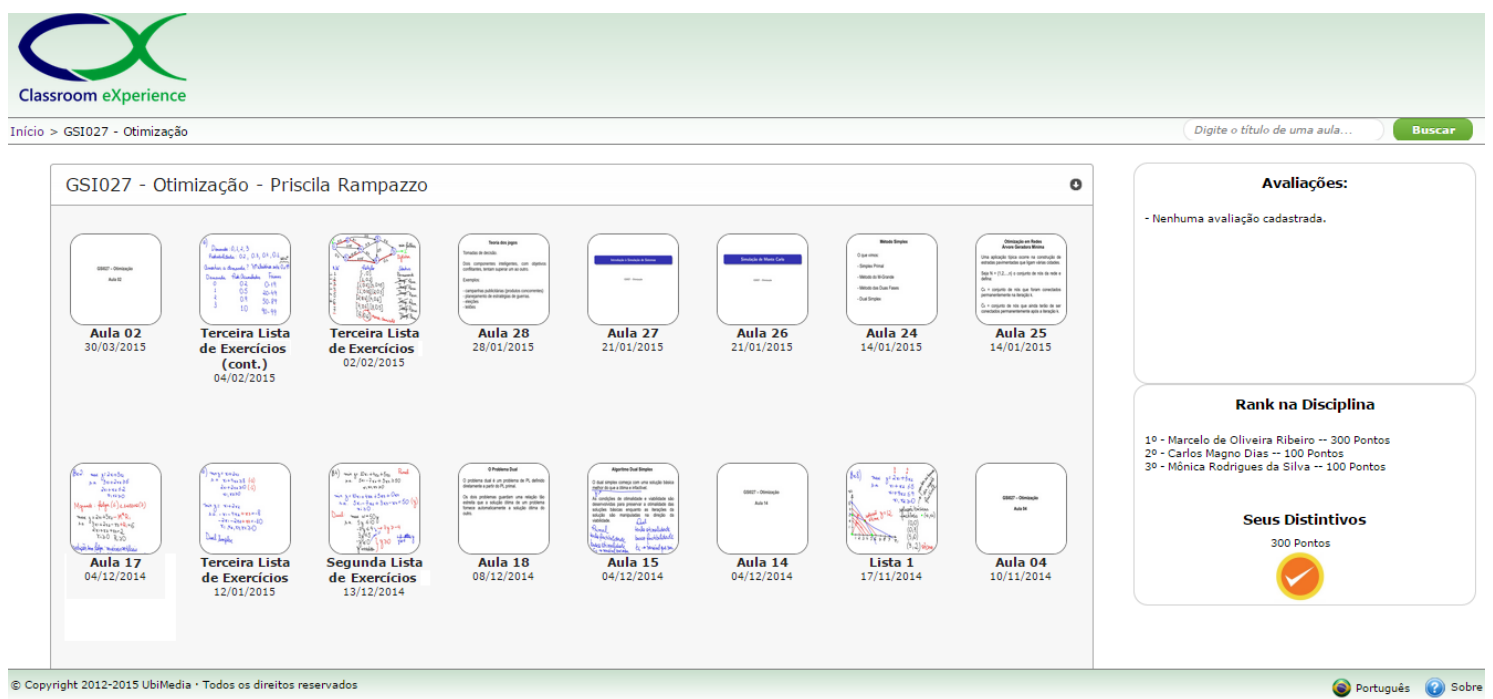

Figura 4. Interface de acesso exibindo o ranking na disciplina e as medalhas já conquistadas.

separação das funcionalidades já existentes no CX, e permitindo que o módulo fosse facilmente integrado ao sistema principal. Para alcançar esse baixo acoplamento, foi definida uma arquitetura RESTful, baseada no padrão REST (Representational State Transfer), para implementação do protótipo. Essa arquitetura proposta utiliza dois componentes principais, que funcionam como mecanismos de comunicação:

- Requisição de Serviços: componente integrado ao CX e responsável por realizar as requisições de serviços através das interações dos usuários com o sistema (como comentários, ranqueamento, respostas aos quizzes, etc), bem como pela exibição das informações sobre as conquistas de cada usuário;

- Processamento das Requisições: formado por serviços Web responsáveis por buscar e armazenar as informações referentes às ações dos usuários.

Os componentes citados acima utilizam uma base de dados relacional para armazenamento das informações. Essa base é formada por quatro tabelas que guardam informações sobre usuários, grupos (disciplinas), símbolos (itens pontuados) e pontuação (histórico de interação pontuada de cada usuário). A base de dados foi construída utilizando PostgreSQL ${ }^{1}$.

Os elementos gráficos da interface foram implementados utilizando folhas de estilo CSS3 (Cascading Style Sheets) ${ }^{2}$ e JavaScript ${ }^{3}$, tecnologias de interpretação padronizadas pelos principais navegadores Web. O código JavaScript contém funções que fazem chamadas a serviços disponibilizados pelo componente responsável por gerenciar o processamento das requisições (Componente de Processamento das Requisições). As requisições são realizadas utilizando a tecnologia Ajax (Asynchronous Javascript and $X M L)^{4}$ e o framework jQuery $1.11^{5}$.

\footnotetext{
${ }^{1} \mathrm{http}: / / w w w . p o s t g r e s q l . o r g /$

${ }^{2}$ http://www.w3.org/Style/CSS/

${ }^{3}$ http://www.w3.org/standards/webdesign/script

${ }^{4}$ http://api.jquery.com/category/ajax/

${ }^{5}$ https://jquery.com/
} 
No componente responsável por realizar o gerenciamento das requisições, foram desenvolvidos serviços Web responsáveis por acessar a base de dados e realizar tarefas de registro e recuperação de informações, tais como a definição e atribuição dos pontos a determinado estudante, a recuperação do rank dos melhores estudantes em uma determinada disciplina e o cálculo do total de pontos de um determinado estudante. Os serviços Web produzem objetos no formato JSON (JavaScript Object Notation) ${ }^{6}$. Dessa forma, os serviços são capazes de se comunicar facilmente com interpretadores JavaScript. Os serviços Web são executados em um servidor Web Tomcat ${ }^{7}$.

A Figura 5 apresenta a integração do módulo de gamificação com o CX. A esquerda da figura é possível observar os componentes Registra Pontuação e Apresenta Rank disponíveis no CX, responsáveis por gravar e buscar informações referentes aos pontos dos usuários. A direita é possível observar a estrutura do módulo de gamificação e como é realizada a comunicação entre seus principais componentes: documento JavaScript, serviços Web, e repositório de dados.

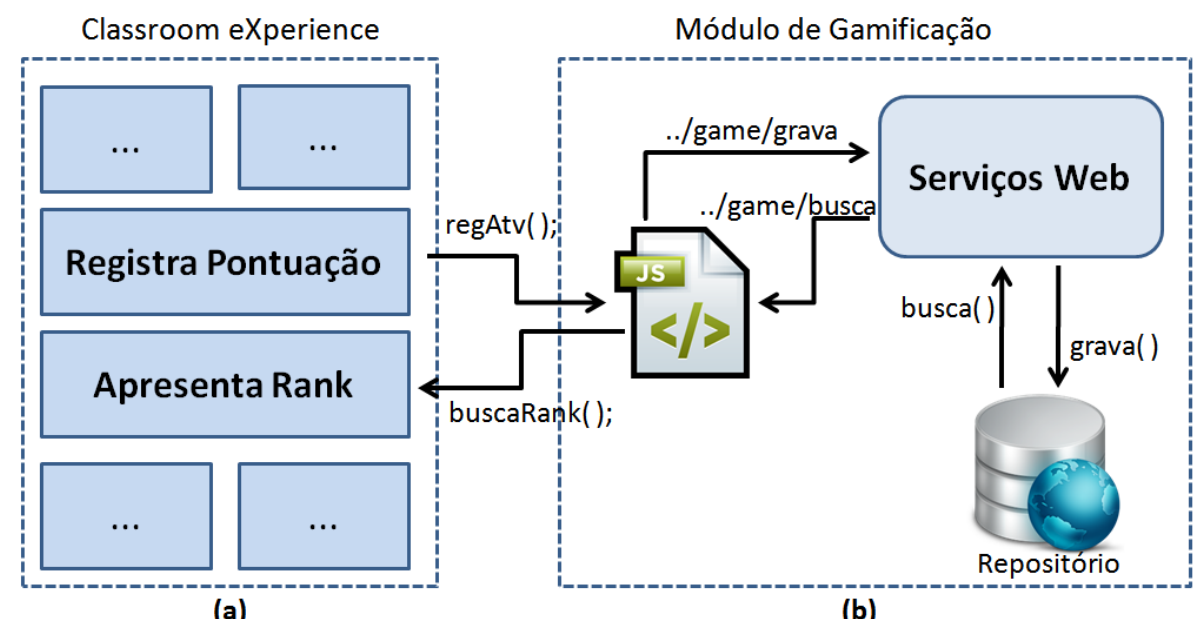

(a)

(b)

Figura 5. Integração do CX ao módulo de gamificação: (a) componentes do CX que se comunicam com o módulo de gamificação; (b) estrutura do módulo de gamificação.

\section{Trabalhos Relacionados}

$\mathrm{Na}$ literatura é possível encontrar diversos trabalhos que exploram o paradigma de computação ubíqua aplicado a ambientes educacionais [Dickson et al. 2012, Diaz Arancibia e Rusu 2014, Gilman et al. 2015]. No entanto, a maioria desses trabalhos tem o foco no desenvolvimento de arquiteturas que disponibilizam conteúdo a seus usuários sem a preocupação com o engajamento dos mesmos. Em outros casos, são explorados recursos para envolver e atrair o usuário, mas nem todos possuem características de AEUs [França e Reategui 2013, Andrade e Canese 2013, Slegers et al. 2015].

Em [França e Reategui 2013], é apresentado o SMILE-BR, uma arquitetura que utiliza conceitos de gamificação em um ambiente de aprendizado baseado em questionamento, a qual procura realizar o engajamento dos estudantes por meio de elementos

\footnotetext{
${ }^{6} \mathrm{http}: / /$ json.org/

${ }^{7}$ http://tomcat.apache.org/
} 
de jogos e também armazenando dados na Web para possibilitar o acesso dos instrutores e estudantes ao projeto a partir da internet. Como na proposta aqui apresentada, o SMILE-BR utiliza elementos de jogos para motivar os estudantes. O SMILE-BR difere da proposta apresentada neste artigo por não ser um ambiente caracterizado como AEU, pois não reproduz de maneira fidedigna as interações provenientes de uma sala de aula. A proposta aqui apresentada vai além da disponibilizada no SMILE-BR, pois além de possibilitar uma interação entre instrutor e estudante, o estudante consegue ainda rever todo o material anteriormente apresentado pelo instrutor, além de criar seus próprios questionamentos (visíveis ao instrutor e a outros estudantes) permitindo, assim, a extensão de todo o conteúdo apresentado.

Alguns trabalhos encontrados na literatura diferem da proposta ora apresentada por serem limitados a determinado domínio, tema ou disciplina [Khaleel et al. 2015, Andrade e Canese 2013, Fernandes e Castro 2013]. Em [Khaleel et al. 2015], é apresentado um ambiente gamificado para cursos de linguagens de programação. Sua arquitetura é dividida em três níveis: material para linguagem Java, elementos de gamificação e projeto de interface. O nível que aborda gamificação trabalha com exercícios/soluções, exames, sistemas de pontuação, ranking, dashboard e relatórios. Esses elementos estimulam o engajamento dos estudantes na interface. Com relação ao contexto de aplicação e diferentemente da arquitetura proposta por Khaleel, a arquitetura aqui apresentada não se limita a uma determinada disciplina, sendo passível de aplicação em qualquer ambiente educacional.

Em [Andrade e Canese 2013], é apresentado o $\exists$ lementar, um sistema aplicado em ambiente de ensino de lógica que incorpora um assistente de provas em um contexto de gamificação. O sistema proposto busca atingir duas dimensões do ponto de vista de interações sociais: atuar como veículo, possibilitando as interações sociais entre seus usuários, e atuar de modo a despertar nos usuários o sentimento de necessidade de interagir com o sistema, sendo assim, parte de um círculo social. Semelhante à proposta aqui apresentada, o $\exists$ lementar cria a ideia de uma rede social, onde estudantes podem interagir com outros usuários do sistema. No entanto, os estudantes não conseguem estender o conteúdo já criado por outros estudantes ou pelo instrutor - uma característica importante disponível na arquitetura apresentada. Outra característica importante da proposta aqui apresentada que o distingue do sistema Đlementar é que, além de ser um ambiente não restrito a determinada disciplina, existe a possibilidade de gerenciamento de turmas, onde o instrutor tem total domínio sobre as atividades de todos os usuários matriculados.

Ainda sobre ambientes educacionais que utilizam gamificação voltada para domínios específicos, Fernandes e Castro apresentam um ambiente educacional gamificado para o ensino da química orgânica [Fernandes e Castro 2013]. O objetivo desta ferramenta é utilizar a gamificação para criar um ambiente onde os estudantes consigam ter acesso a conteúdos de apoio ao aprendizado e sejam estimulados a cumprir tarefas para facilitar a fixação desse conteúdo. O sistema exige que o instrutor disponibilize os jogos, o pode ser considerada uma limitação, pois muitas vezes os instrutores não possuem domínio na criação desses recursos. Diferente da proposta aqui apresentada, o sistema não cria mecanismos para interação e colaboração entre os usuários, nem utiliza recursos como medalhas, conquistas ou pontos, limitando-se apenas a um quadro de líderes. 


\section{Conclusões}

Gamificação é um tema de pesquisa que tem atraído a atenção de um grande número de pesquisadores e profissionais. Inspirada na experiência do usuário de jogos de computador, gamificação explora a ideia de se usar elementos de jogos em diferentes contextos. Estudos mostram que a gamificação tem o potencial de melhorar consideravelmente a experiência e o engajamento dos usuários em uma variedade de domínios de aplicação, incluindo ambientes educacionais [Amriani et al. 2013]. O presente trabalho apresentou o processo de concepção e desenvolvimento de um modelo para integração de conceitos de gamificação a Ambientes Educacionais Ubíquos. O modelo foi implementado como um módulo de software com arquitetura fracamente acoplada e então integrado a um sistema educacional real que está em pleno uso. O módulo estende a interface de acesso desse sistema, registrando as interações dos estudantes durante sua utilização para, com isso, gerar diferentes rankings de pontuação entre eles e outros mecanismos de gamificação.

As informações produzidas pelos usuários a partir das atividades de colaboração do CX - anotações e ranqueamento - além de enriquecerem o conteúdo originalmente capturado e fomentarem a aprendizagem coletiva por meio de uma rede social, geram subsídios para requisitos de recomendação e personalização a partir do próprio conteúdo gerado pela população de usuários da plataforma (estudantes e instrutores). A proposta ora apresentada visou complementar esse enriquecimento, permitindo que os conceitos de gamificação empregados contribuíssem para o engajamento dos usuários na utilização da plataforma e para o compartilhamento e a extensão de seu conteúdo. Essa dinâmica de competição cria um ambiente mais interativo e atraente, promovendo assim, um maior interesse por parte dos estudantes. Ao proporcionar novas interações sobre o ambiente do estudante, o módulo de gamificação atua também no ambiente do instrutor, permitindo a verificação de desempenho dos estudantes quanto à utilização da dinâmica e dos mecanismos de jogos.

Como trabalhos futuros, planeja-se a realização de experimentos para validar a eficácia e usabilidade do protótipo desenvolvido, a criação de arquiteturas para recomendação de conteúdo baseados nos dados de utilização do ambiente fornecidas pelo módulo de gamificação, e a utilização dos dados gerados pelo sistema de gamificação para auxiliar na identificação do estilo de aprendizado do estudante.

\section{Agradecimentos}

Os autores agradecem a CAPES, CNPq, FAPEMIG e PET/SESu/MEC pelo apoio.

\section{Referências}

Abowd, G. D. e Mynatt, E. D. (2000). Charting past, present, and future research in ubiquitous computing. ACM Trans. on Computer-Human Interaction, 7(1):29-58.

Amriani, A., Aji, A. F., Utomo, A. Y., e Junus, K. M. (2013). An empirical study of gamification impact on e-learning environment. In Proc. of ICCSNT, p. 265-269.

Andrade, J. O. e Canese, M. (2013). Um sistemaweb gamificado para a aprendizagem de lógica formal. In Proc. of SBIE.

Araujo, R., Brant-Ribeiro, T., Cattelan, R., De Amo, S., e Ferreira, H. (2013). Personalization of interactive digital media in ubiquitous educational environments. In Proc. of SMC, p. 3955-3960. 
Deterding, S., Dixon, D., Khaled, R., e Nacke, L. (2011). From game design elements to gamefulness: Defining gamification. In Proc. of MindTrek, p. 9-15.

Diaz Arancibia, J. e Rusu, C. (2014). Ubiquitous computer-supported collaborative learning: A literature review. In Proc. of ITNG, p. 593-598.

Dickson, P. E., Warshow, D. I., Goebel, A. C., Roache, C. C., e Adrion, W. R. (2012). Student reactions to classroom lecture capture. In Proc. of ITiCSE, p. 144-149.

Domínguez, A., de Navarrete, J. S., de Marcos, L., Fernández-Sanz, L., Pagés, C., e Martínez-Herráiz, J.-J. (2013). Gamifying learning experiences: Practical implications and outcomes. Computers \& Education, 63(0):380 - 392.

Fernandes, A. M. d. R. e Castro, F. S. (2013). Ambiente de ensino de química orgânica baseado em gamificação. In Proc. of SBIE.

Ferreira, H. N. M., Araújo, R. D., de Amo, S., e Cattelan, R. G. (2012). Classroom Experience: A Platform for Multimedia Capture and Access in Instrumented Educational Environments. In Proc. of SBSC, p. 59-64.

França, R. M. e Reategui, E. B. (2013). Smile-br: aplicação de conceitos de gamificação em um ambiente de aprendizagem baseado em questionamento. In Proc. of SBIE.

Gilman, E., Sanchez Milara, I., Cortes, M., e Riekki, J. (2015). Towards user support in ubiquitous learning systems. Trans. on Learning Technologies, 8(1):55-68.

Khaleel, F. L., Ashaari, N. S., Meriam, T. S., Wook, T., e Ismail, A. (2015). The study of gamification application architecture for programming language course. In Proc. of IMCOM, p. 17:1-17:5.

Kientz, J. A. (2012). Embedded capture and access: encouraging recording and reviewing of data in the caregiving domain. Personal Ubiquitous Comput., 16(2):209-221.

Kiili, K., de Freitas, S., Arnab, S., e Lainema, T. (2012). The design principles for flow experience in educational games. Procedia Computer Science, 15(0):78 - 91.

Lee, J. J. e Hammer, J. (2011). Gamification in education: What, how, why bother? Academic Exchange Quarterly, 15(2):2.

Mendonca, I. E. S., Araujo, R., Mendes, M. M., Brant-Ribeiro, T., A., D. F., e Cattelan, R. (2014). Explorando funcionalidades sociais e colaborativas em ambientes educacionais ubíquos. In Proc. of SBIE.

Seaborn, K. e Fels., D. I. (2014). Gamification in theory and action: A survey. International Journal of Human-Computer Studies, 74:14-31.

Settle, A., Dettori, L., e Davidson, M. J. (2011). Does Lecture Capture Make a Difference for Students in Traditional Classrooms. In Proc. of ITICSE, p. 78-82.

Slegers, K., Ruelens, S., Vissers, J., e Duysburgh, P. (2015). Using game principles in ux research: A board game for eliciting future user needs. In Proc. of CHI, p. 1225-1228.

Truong, K. N. e Hayes, G. R. (2009). Ubiquitous Computing for Capture and Access. Foundations and Trends in Human-Computer Interaction, 2(2):95-171.

Weiser, M. (1991). The Computer for the 21st Century. Scient. American, 265(3):66-75. 